\title{
Phase Coherence and Andreev Reflection in Topological Insulator Devices
}

\author{
A. D. K. Finck, ${ }^{1}$ C. Kurter, ${ }^{1}$ Y. S. Hor, ${ }^{2}$ and D. J. Van Harlingen ${ }^{1}$ \\ ${ }^{1}$ Department of Physics and Materials Research Laboratory, University of Illinois at Urbana-Champaign, \\ Urbana, Illinois 61801, USA \\ ${ }^{2}$ Department of Physics, Missouri University of Science and Technology, Rolla, Missouri 65409, USA
}

(Received 3 June 2014; published 4 November 2014)

\begin{abstract}
Topological insulators (TIs) have attracted immense interest because they host helical surface states. Protected by time-reversal symmetry, they are robust to nonmagnetic disorder. When superconductivity is induced in these helical states, they are predicted to emulate $p$-wave pairing symmetry, with Majorana states bound to vortices. Majorana bound states possess non-Abelian exchange statistics that can be probed through interferometry. Here, we take a significant step towards Majorana interferometry by observing pronounced Fabry-Pérot oscillations in a TI sandwiched between a superconducting and a normal lead. For energies below the superconducting gap, we observe a doubling in the frequency of the oscillations, arising from an additional phase from Andreev reflection. When a magnetic field is applied perpendicular to the TI surface, a number of very sharp and gate-tunable conductance peaks appear at or near zero energy, which has consequences for interpreting spectroscopic probes of Majorana fermions. Our results demonstrate that TIs are a promising platform for exploring phase-coherent transport in a solid-state system.
\end{abstract}

DOI: 10.1103/PhysRevX.4.041022

\section{INTRODUCTION}

When electrons travel far distances without scattering off of impurities, their wavelike nature becomes apparent through signatures of interference. A prominent example is ballistic transmission through a barrier between two semiinfinite leads. Imperfect transmission through the interfaces causes partial reflection of impinging electrons. Within the barrier, constructive interference of reflected waves leads to periodic modulation of total transmission through the barrier, resulting in resonant transmission whenever the electron wavelength is an integer multiple of twice the barrier length. This is equivalent to $k_{F} L=\pi n$, where $k_{F}$ is the Fermi wave vector, $L$ is the barrier length, and $n$ is a nonzero integer.

This quantum phenomenon, known as Fabry-Pérot interference due to its similarity to the eponymous classical optical effect, has been observed in a variety of mesoscopic systems, including nanotubes [1], nanowires [2], and graphene [3]. In topological systems with protected edge states [4], interferometry takes on a new significance as particles traveling through the edge states can acquire an additional phase if their paths enclose exotic quasiparticles known as anyons [5]. A class of anyons known as Majorana

Published by the American Physical Society under the terms of the Creative Commons Attribution 3.0 License. Further distribution of this work must maintain attribution to the author(s) and the published article's title, journal citation, and DOI.
Subject Areas: Condensed Matter Physics,

Superconductivity,

Topological Insulators

bound states (MBSs) can be realized in a topological insulator (TI) with induced superconductivity [6-8]. MBSs can encode quantum information nonlocally, realizing topologically protected qubits whose states can be read out through interferometry [9-11]. Recently, there has been much experimental progress in realizing superconductivity in topological systems through the study of Josephson junctions constructed on the surface of various 3D TIs [12-21].

In this paper, we describe direct studies of phase coherence and Andreev reflection in TI devices. In each of our samples, a thin flake of the $3 \mathrm{D} \mathrm{TI} \mathrm{Bi}_{2} \mathrm{Se}_{3}$ is placed in contact with both a superconducting lead and a normal metal lead. The contacts are highly transparent, permitting Andreev reflection between the $\mathrm{Bi}_{2} \mathrm{Se}_{3}$ and the superconducting lead. The chemical potential of the $\mathrm{Bi}_{2} \mathrm{Se}_{3}$ is tuned with electrostatic gating. In more resistive devices, we find evidence of Fabry-Pérot oscillations, demonstrating gate-tunable, phase-coherent transport in a TI. For source-drain biases below the energy gap of the superconducting lead, we observe a doubling of the frequency of the conductance oscillations. This provides firm evidence of de Gennes-Saint-James resonances [22,23], in which an additional phase accumulated from Andreevreflected holes changes the period of geometric resonances by a factor of 2 . When a large magnetic field is applied perpendicular to the TI surface, an intricate set of lowenergy resonances emerges. These features include zeroenergy peaks that can persist for a range of chemical 
potential and magnetic field, sharing many properties of anomalies observed in nanowires [24-28]. Although such anomalies were interpreted in terms of MBSs, the coexistence of the low-energy resonances with phase-coherent signatures at high magnetic fields in our devices suggests that alternative explanations are possible, including weak antilocalization [29]. Our results encourage further studies of gate-tunable phase coherence in $\mathrm{Bi}_{2} \mathrm{Se}_{3}$ in order to perform interferometric searches for MBSs. For example, it is necessary to discern what role topological surface states play in the Fabry-Pérot oscillations.

\section{EXPERIMENTAL METHODS}

Single crystals of $\mathrm{Bi}_{2} \mathrm{Se}_{3}$ are grown by melting a mixture of pure $\mathrm{Bi}$ and $\mathrm{Se}$ in a stoichiometric ratio of $1.9975: 3$ (Bi:Se) in a vacuum quartz tube at $800^{\circ} \mathrm{C}$. Thin flakes (7-20 nm) of $\mathrm{Bi}_{2} \mathrm{Se}_{3}$ are exfoliated onto silicon substrates covered by a $300 \mathrm{~nm}$ thick $\mathrm{SiO}_{2}$ layer. Such thin flakes typically have a $2 \mathrm{D}$ carrier density of $N_{2 \mathrm{D}} \approx 10^{13}-10^{14} \mathrm{~cm}^{-2}$ and low temperature mobility $\mu \approx 10^{2}-10^{3} \mathrm{~cm}^{2} / \mathrm{V} \mathrm{s}$, as determined from measurements of separate Hall bar devices with similar thicknesses as the devices mentioned in the main section and Supplemental Material [30]. For estimates of the Fermi velocity, we use the value $v_{F}=4.2 \times 10^{5} \mathrm{~m} / \mathrm{s}$ from angle-resolved photoemission spectroscopy measurements of similar crystals of $\mathrm{Bi}_{2} \mathrm{Se}_{3}$ [31]. Weak antilocalization measurements of the Hall bar devices give typical phase-coherence lengths of $\ell_{\phi}=300-1000 \mathrm{~nm}$ at $10 \mathrm{mK}$. For the Andreev reflection devices, superconducting leads are defined by conventional $e$-beam lithography and a subsequent dc sputtering of $50 \mathrm{~nm}$ of $\mathrm{Nb}$ at room temperature. Tunnel junctions of $\mathrm{Au} / \mathrm{Al}_{2} \mathrm{O}_{3} / \mathrm{Nb}$ reveal a superconducting gap of $\Delta=1.5 \mathrm{meV}$ for our $\mathrm{Nb}$ films immediately after sputtering; in top-gated TI-Nb devices, the inverse proximity effect and additional nanofabrication processing will likely reduce the gap below this pristine value. Normal metal leads are deposited through $e$-beam evaporation of $5 \mathrm{~nm}$ of Ti and $50 \mathrm{~nm}$ of Au. Brief Ar ion milling is employed before metallization in situ to ensure good contact between the $\mathrm{Bi}_{2} \mathrm{Se}_{3}$ and the leads. This process yields reliably transparent contacts, as demonstrated by the consistent observation of supercurrents in topological insulator Josephson junctions $[16,18,19,21]$. Contact resistance is estimated to be much less than $100 \Omega$ and is thus negligible. Typical Nb-Au lead separation is $100-250 \mathrm{~nm}$. Applying a bias to the silicon substrate permits backgating. A top gate is created by covering the sample with $30 \mathrm{~nm}$ of alumina via atomic layer deposition and deposition of $\mathrm{Ti} / \mathrm{Au}$ over the exposed $\mathrm{Bi}_{2} \mathrm{Se}_{3}$. The device discussed in the main section is covered with alumina but lacks a top gate. The devices are thermally anchored to the mixing chamber of a cryogen-free dilution refrigerator equipped with a vector magnet and filtered wiring. We perform lowfrequency transport measurements with standard lock-in techniques, typically with a $10 \mathrm{nA}$ ac excitation at $f=73 \mathrm{~Hz}$. Unless stated otherwise, all measurements are performed at a base mixing chamber temperature of $20 \mathrm{mK}$.

\section{TRANSPORT IN ZERO FIELD}

\section{A. Andreev reflection and reentrant resistance effect}

We study Andreev reflection in thin $(\sim 10 \mathrm{~nm})$ flakes of the $3 \mathrm{D}$ topological insulator $\mathrm{Bi}_{2} \mathrm{Se}_{3}$ connected to both superconducting and normal metal leads separated by $L=100-250 \mathrm{~nm}$. An example of a device with $L=$ $230 \mathrm{~nm}$ is pictured in Fig. 1(a). Either top or back gates are used to tune the chemical potential of the surface states, depicted in Fig. 1(b). Here, we focus on one sample, with qualitatively similar results obtained from others [30]. At low temperature, we observe an enhancement in conductance at source-drain biases $(V)$ below the niobium gap, as expected for Andreev reflection [Fig. 2(a)] [32-34]. Near $V=0$, however, there is a downturn in the conductance. This is consistent with the reentrant resistance effect $[35,36]$, in which Andreev reflection is canceled out by the proximity effect in the limit of zero energy and zero temperature [36,37]. Maximum conductance occurs at either a characteristic temperature or $V$ that is governed by the Thouless energy $E_{T}=\hbar D / L^{2}$, where $D$ is the diffusion constant and $L$ is the separation between the superconducting and normal metal leads on the bismuth selenide. Magnetotransport and angle-resolved photoemission spectroscopy measurements [31] allow us to calculate $E_{T}$ through $D=\frac{1}{2} v_{F} l=\frac{1}{2} v_{F} \mu(h / 2 e) \sqrt{N_{2 D} / \pi} \approx 0.0025 \mathrm{~m}^{2} / \mathrm{s}$, where $v_{F}$ is the Fermi velocity, $l$ is the mean-free path, $\mu$ is the mobility, and $N_{2 \mathrm{D}}$ is the $2 \mathrm{D}$ carrier density. This results (a)

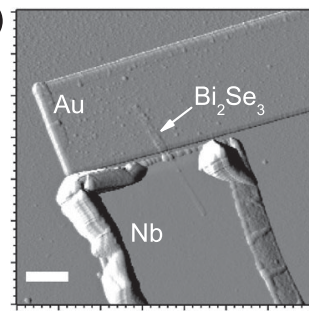

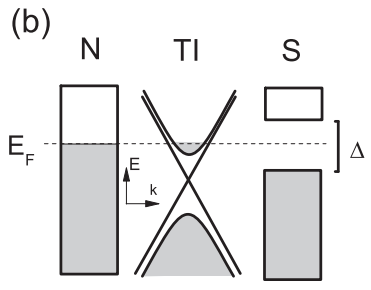

FIG. 1. (a) AFM image of N-TI-S device, where $\mathrm{N}$ stands for the normal metal lead $(\mathrm{Au})$ and $\mathrm{S}$ is the superconducting lead $(\mathrm{Nb})$. The $\mathrm{Bi}_{2} \mathrm{Se}_{3}$ segment originally consisted of two adjacent nanoribbons that are each $9 \mathrm{~nm}$ thick, but subsequent AFM imaging after measurements revealed only one nanoribbon remaining under the niobium lead [30]. Separation between $\mathrm{N}$ and $\mathrm{S}$ leads is $L=230 \mathrm{~nm}$, as determined from SEM. White scale bar is $1 \mu \mathrm{m}$. (b) Energy diagram for the three materials in the device. The TI (whose dispersion relation is depicted with both gapless helical surface states and gapped trivial states) is sandwiched between a compressible normal metal and a superconductor with an energy gap $\Delta$. 
in $E_{T}=30 \mu \mathrm{V}$ for $L=230 \mathrm{~nm}$, consistent with our transport measurements $\left(T_{\max }=900 \mathrm{mK}\right.$ and $\left.V_{\max }=85 \mu \mathrm{V}\right)$. At high temperature [Fig. 2(b)], the conductance dip disappears and only a broad peak from Andreev reflection remains. We note in passing a remarkable similarity between Fig. 2(a) and the predictions of Ref. [38], which posited that anomalous Andreev bound states on the surface of a topological superconductor would generate an asymmetric zero-bias tunneling conductance peak [39] that splits at low temperature. However, we feel that our data are most likely a result of the reentrant resistance effect. Devices with smaller $L$ possess larger $E_{T}$ and are
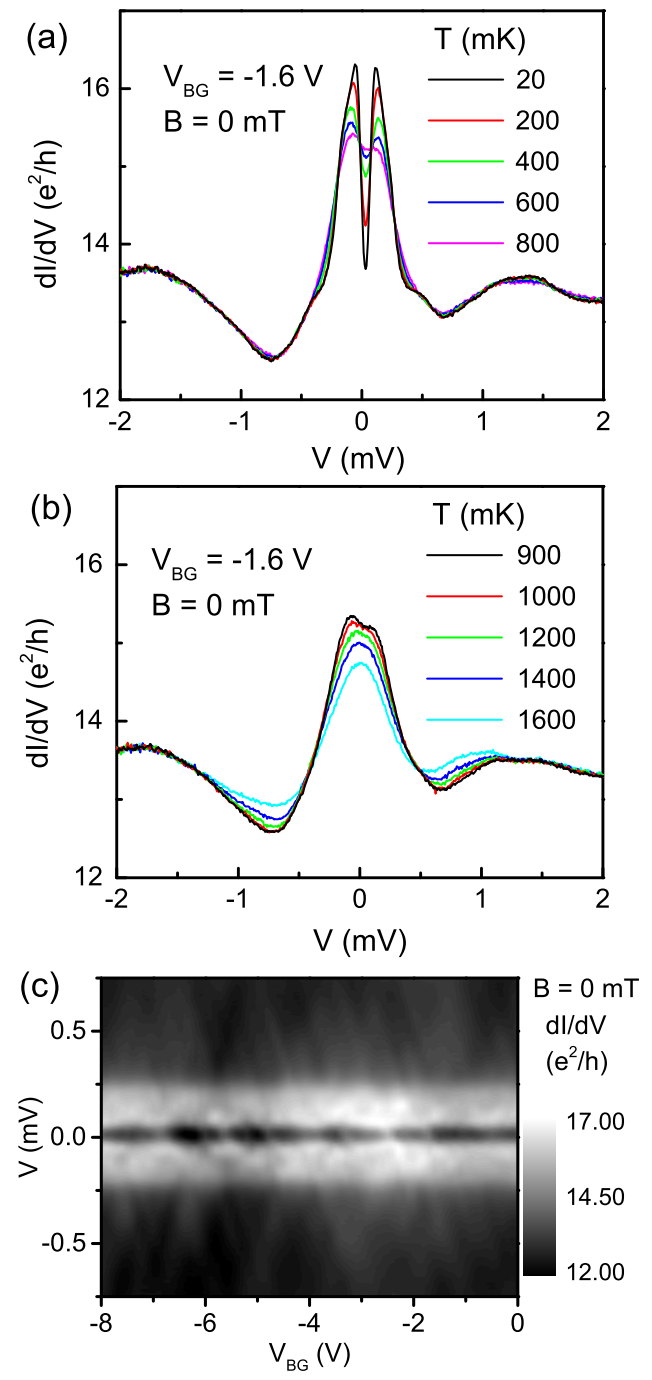

FIG. 2. (a) Conductance versus source-drain bias $V$ for various temperatures at zero field. At low bias, the conductance is suppressed due to the reentrant resistance effect. (b) Conductance traces at zero field and elevated temperature. When the temperature exceeds the Thouless energy, the low-bias conductance suppression is replaced by a broad conductance peak. (c) Color plot of $d I / d V$ versus source-drain bias $V$ and backgate bias $V_{\mathrm{BG}}$ at zero field and $20 \mathrm{mK}$. observed to have a broader suppression in conductance around zero bias [30].

\section{B. Fabry-Pérot oscillations}

To demonstrate phase-coherent transport, we turn to gate-tuned measurements. In Fig. 2(c), we display a color plot of conductance versus $V$ and backgate voltage $V_{\mathrm{BG}}$. At higher bias [Fig. 3(a)], we observe a checkerboard pattern that is characteristic of Fabry-Pérot oscillations when there is a finite probability of backscattering at the interfaces
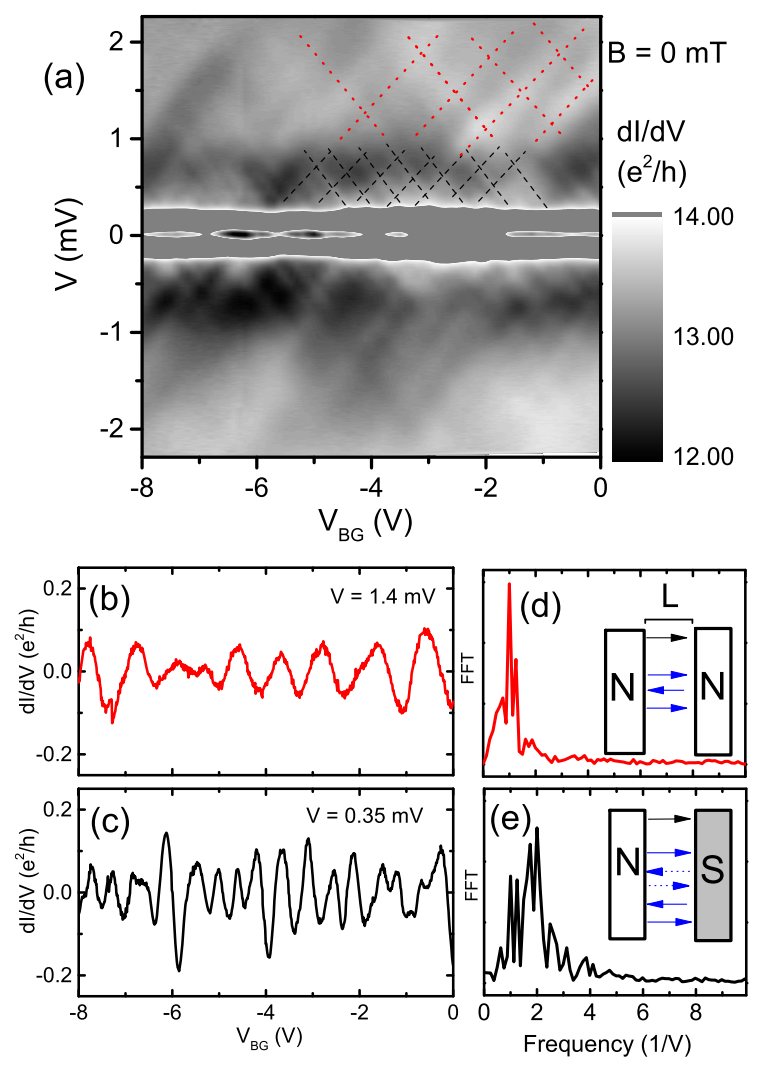

FIG. 3. (a) Color plot of $d I / d V$ versus source-drain bias $V$ and backgate bias $V_{\mathrm{BG}}$ accentuating the high-bias features. Red dotted lines are guides to the eye to indicate the checkerboard pattern for conventional Fabry-Pérot oscillation at high bias. Black dashed lines follow the low-bias features for de GennesSaint-James resonances. (b),(c) Plots of differential conductance versus backgate bias for two different values of source-drain bias $V$; in both cases, we subtract a slowly varying background. Panel (b) shows the Fabry-Pérot oscillations at high bias, with period $\Delta V_{\mathrm{BG}} \approx 1 \mathrm{~V}$; corresponding FFT is shown in (d). Panel (c) shows the low-energy de Gennes-Saint-James resonances with period $\Delta V_{\mathrm{BG}} \approx 0.5 \mathrm{~V}$; corresponding FFT is shown in (e). The illustrations in insets of (d) and (e) symbolize the two sets of particle trajectories for Fabry-Pérot resonances (with electrons only) and de Gennes-Saint-James resonances (mixture of holes and electrons), respectively. Solid arrows represent the trajectories of electrons while dashed arrows represent those of holes. Transmitted particles are shown in black while reflected particles are shown in blue. 
between the topological insulator segment and the metallic leads. Such backscattering could result from either a finite barrier or Fermi velocity mismatch, as well as the mixing of spin states in order to satisfy the spin-momentum locking from strong spin-orbit coupling. The periodic variation in conductance coexists with aperiodic universal conductance fluctuations (UCFs), which have been previously reported in $\mathrm{Bi}_{2} \mathrm{Se}_{3}$ [40]. Whenever the Fermi wave vector $k_{F}$ is tuned to a Fabry-Pérot resonance, constructive interference generates enhanced conductance. Shifts in $V$ or $V_{\mathrm{BG}}$ will shift the Fermi energy $E_{F}$ of incident electrons by a proportional amount. With a linear approximation $E_{F}=\hbar v_{F} k_{F}$, one expects subsequent Fabry-Pérot resonances to differ in energy by $\Delta E=\left(h v_{F} / 2 L\right)$. For $V>\Delta / e$ [Fig. 3(b)], we observe oscillations with period $\Delta E=0.8 \mathrm{mV}$. This disagrees somewhat with the estimate $3.8 \mathrm{mV}$, possibly due to renormalization of the Fermi velocity from electronelectron interactions or strong coupling to a superconductor. We note that while previous studies have provided evidence of phase coherence in topological insulators through weak antilocalization (e.g., Ref. [41]), here we demonstrate direct, gate-tunable signatures of phasecoherent transport. Devices with smaller $L$ are observed to have larger $\Delta E$ [30]. Remarkably, such oscillations can be observed without special care for cleanliness, as achieved in other materials through current annealing or isolating the material from dielectric impurities by suspension from the substrate $[2,42]$. This is likely due to the topological protection of the helical surface states [4], although we do not rule out the contribution from trivial states [43]. The peak-to-peak amplitude of the oscillations are $\approx 0.2 e^{2} / h$, independent of normal state resistance, which varies from $600 \Omega$ to $21 \mathrm{k} \Omega$ in our three devices. This suggests that while bulk carriers might contribute to the zero-bias conductance, the quantum interference is generated by a fixed number of $2 \mathrm{D}$ channels from the surface states, whose transport contributions are expected to be largely independent of bulk conditions [44].

\section{Interplay between geometric resonances and Andreev reflection}

The most unusual feature of the Fabry-Pérot oscillations in Fig. 3 is a doubling of their frequency when $V$ is less than the superconducting gap of niobium, as shown in Fig. 3(c). Fourier transforms of the oscillations [Figs. 3(d) and 3(e)] confirm this frequency doubling via a shift in spectral weight from $\Delta V_{\mathrm{BG}}=1 \mathrm{~V}$ to $\Delta V_{\mathrm{BG}}=0.5 \mathrm{~V}$. Coulomb repulsion can split otherwise degenerate levels [45], but the high degree of coupling between the leads and the TI should minimize charging effects. Furthermore, the peaks in conductance at low bias are evenly separated in $V_{\mathrm{BG}}$, unlike the uneven spacing expected for split degeneracies. Instead, this behavior can be understood as a modification of phase-coherent transport by Andreev reflection. Lowenergy electrons passing from the cavity to the superconducting lead will be retroreflected as a hole with high probability in the absence of significant barrier, depicted in the illustration in the inset of Fig. 3(e). As first pointed out by de Gennes and Saint-James, the reflected hole traverses the cavity and reflects off the interface with the normal metal lead. The holes do not interfere with the incident electrons; instead, the hole returns to the superconducting lead to undergo Andreev reflection again, leading to an emitted electron. This reflected electron can traverse the cavity and subsequently interfere with incident electrons. Because in this process the reflected particles travel four times the cavity length rather than twice the cavity length, the corresponding geometric resonances occur with twice the frequency. Although this interplay between Andreev reflection and geometric resonances was predicted over 50 years ago [22,23], until recently there has only been indirect evidence of this effect in the form of low-energy conductance peaks [46,47] without any chemical potential tuning or associated observation of high-energy resonances corresponding to FabryPérot oscillations, which would identify them as geometric resonances. Here, we observe a clear crossover from high-frequency geometric resonances below the energy gap to low-frequency resonances above the gap, thus providing unambiguous evidence of de Gennes-SaintJames resonances.

In Fig. 4(a), we explore the temperature dependence of the de Gennes-Saint-James oscillations. The amplitude of the oscillations is rapidly suppressed by thermal fluctuations, decreasing by roughly a factor of 2 between $20 \mathrm{mK}$ and $1.6 \mathrm{~K}$. A similar suppression is observed for the Fabry-Pérot oscillations at high source-drain bias [Fig. 4(b)]. We see in Fig. 4(c) that the amplitude of the oscillations shows an exponential decay with temperature (i.e., $\Delta G \approx e^{-T}$ ), consistent with a dephasing length that scales with $T^{-1}$ (assuming $\Delta G \approx e^{-L / L_{\phi}}$ ). This behavior has been previously seen in other open and ballistic mesoscopic systems [48-51], helping to confirm that the observed oscillations are an interference phenomenon. We note that while weak antilocalization measurements in separate Hall bar devices show qualitatively similar sensitivity to temperature [30], these larger devices exhibit $L_{\phi} \approx T^{-0.5}$ [52].

Our observation of Fabry-Pérot oscillations also helps us to understand the behavior of planar Josephson junctions fabricated on the surface of TIs. We have constructed Josephson junctions with similar lead separation on these $\mathrm{Bi}_{2} \mathrm{Se}_{3}$ flakes [19,21], whose critical currents also vanish between 1.6 and $2 \mathrm{~K}$. The critical current for these TI Josephson junctions as well as those from other groups $[15,20]$ were found to rapidly increase with decreasing temperature, which was interpreted to be a sign of ballistic behavior. Our more direct evidence of ballistic transport through Fabry-Pérot oscillations helps to confirm this interpretation. 

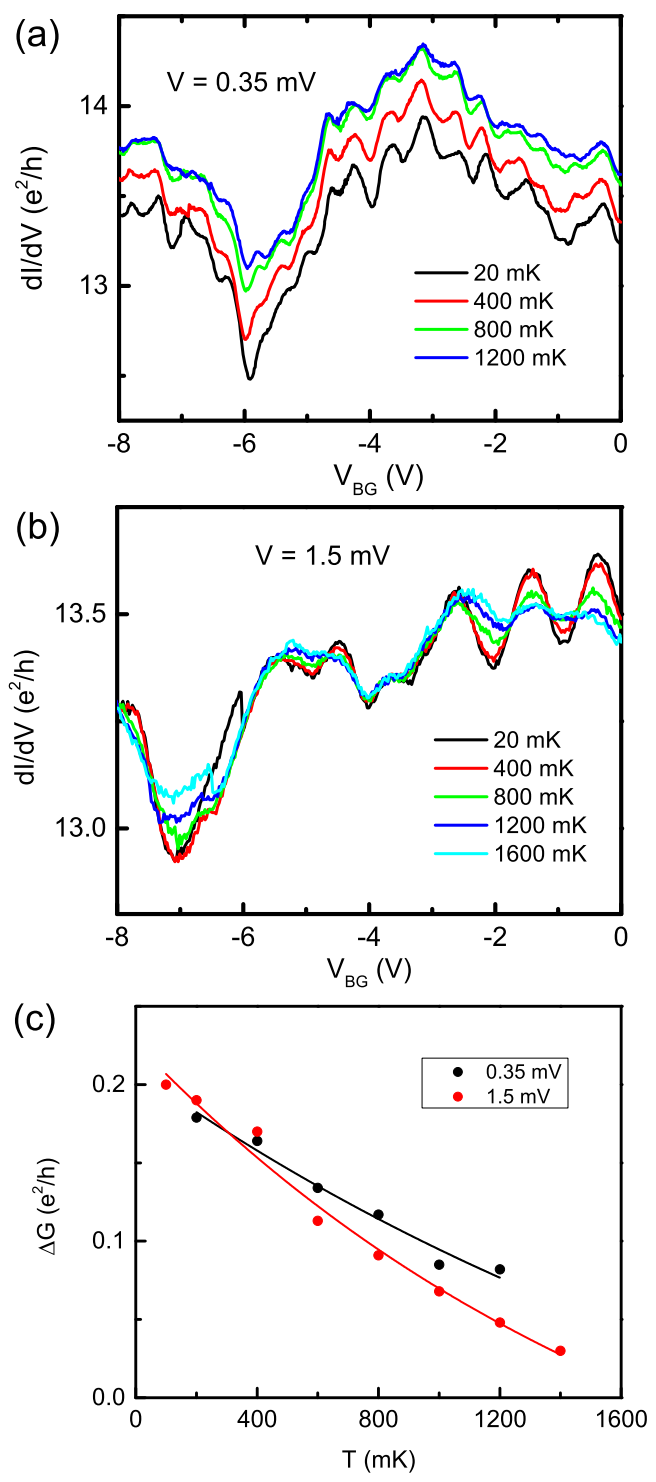

FIG. 4. Temperature dependence of geometric resonances for source-drain bias (a) $V=0.35 \mathrm{mV}$ and (b) $V=1.5 \mathrm{mV}$. (c) Peak-to-peak amplitude $\Delta G$ of geometric resonances versus temperature (circles) for two different source-drain biases, accompanied by exponential fits (solid lines).

We emphasize that none of the transport signatures presented so far are unique to topological systems. Crystals of $\mathrm{Bi}_{2} \mathrm{Se}_{3}$ are known to possess both topological surface states as well as trivial states in the bulk from unintentional doping [4] and at the surface due to band bending [43]. The trivial states can contribute to electrical conductance and potentially exhibit phase coherence like in a conventional semiconductor. Furthermore, there remains the question of the role of the top and bottom topological surface states. We observe only one set of geometric resonances in our devices, independent of whether a bias is applied to the back gate or a top gate [30]. We do not observe any gate-independent resonances that remain at fixed source-drain bias. This is consistent with phasecoherent transport through both upper and lower surface states whose chemical potentials are locked together due to large interlayer electrostatic coupling [53].

\section{PHASE-COHERENT TRANSPORT IN A MAGNETIC FIELD}

We next consider transport in magnetic fields applied perpendicular to the TI surface. While in-plane fields have no influence up to at least $100 \mathrm{mT}$, with out-of-plane fields, we observe a steady evolution of the background signal from universal conductance fluctuations and suppression of superconductivity, as shown in Fig. 5(a) and 5(b). While the amplitude of the Fabry-Pérot oscillations are similarly reduced, their phase does not change appreciably, suggesting that the fields we use are too small to observe the phase shift expected from Klein backscattering [54]. Small shifts can be observed in the de Gennes-Saint-James resonances [Fig. 5(a)], but it is difficult to reliably disentangle them from the evolving background signal due to universal conductance fluctuations.

\section{A. Emergence of low-energy resonances at high field}

Strikingly, in two of our samples, we observe a number of narrow, low-energy conductance peaks emerge
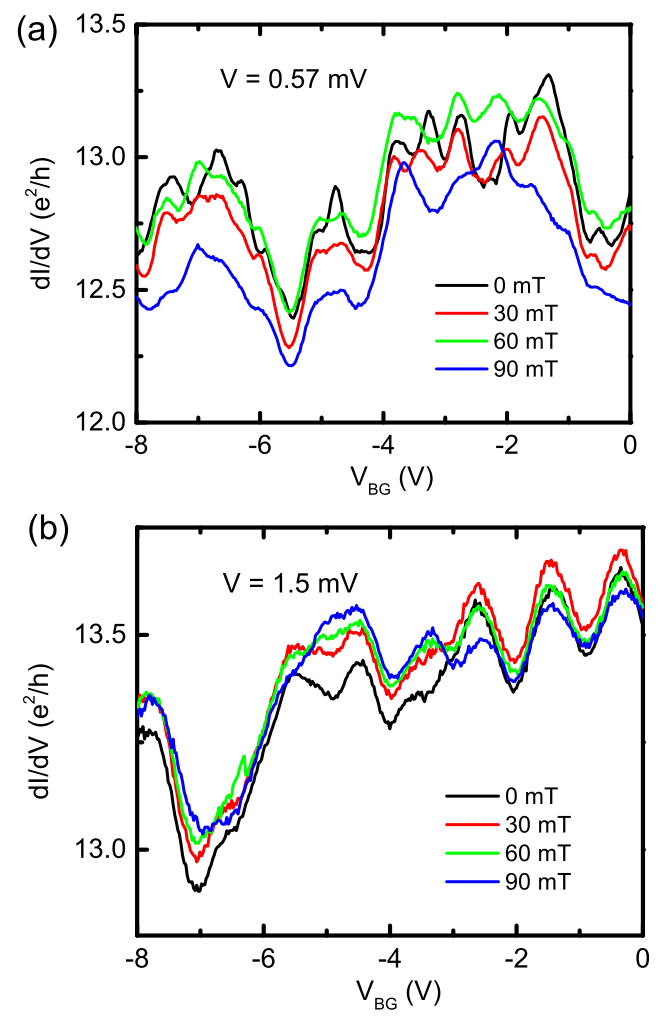

FIG. 5. Magnetic field dependence of geometric resonances for source-drain bias (a) $V=0.35 \mathrm{mV}$ and (b) $V=1.5 \mathrm{mV}$. 

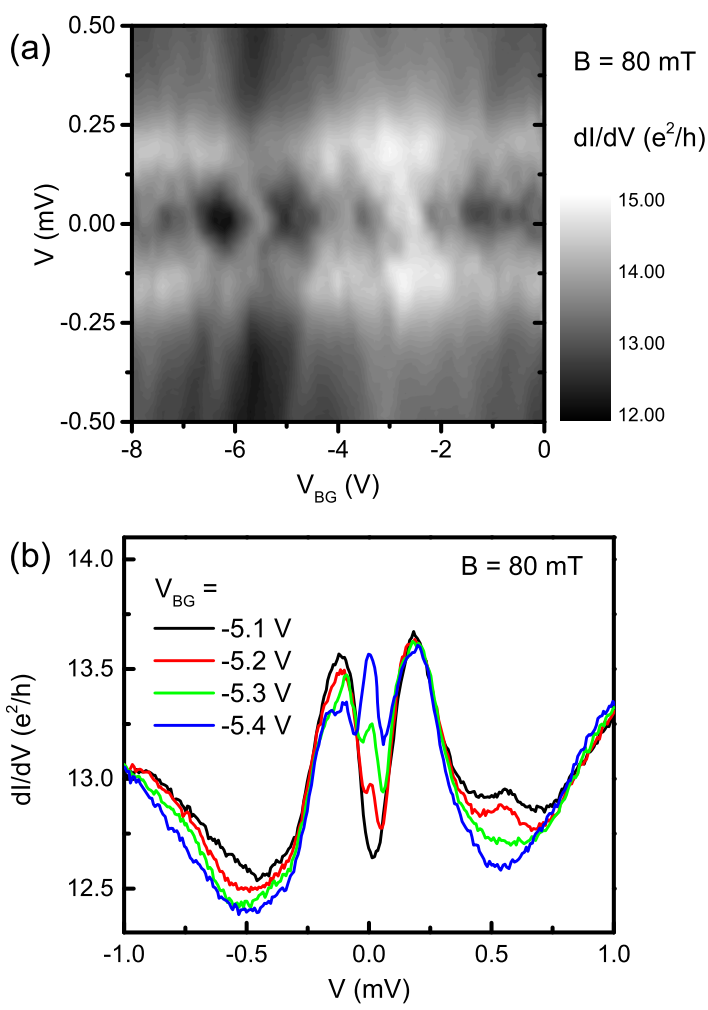

FIG. 6. (a) Color plot of $d I / d V$ versus source-drain bias $V$ and backgate bias $V_{\mathrm{BG}}$ at $\mathrm{B}=80 \mathrm{mT}$, with intricate low-energy resonances. (b) Example conductance traces at $80 \mathrm{mT}$, showing evolution of zero-bias peak near $V_{\mathrm{BG}}=-5.4 \mathrm{~V}$.

at large magnetic fields. An example is shown in Fig. 6(a), with data from the second device shown in the Supplemental Material [30]. The location of the peaks can be tuned readily with $V_{\mathrm{BG}}$, causing them to even form a zero-bias conductance peak for certain gate ranges with height $\approx 0.2 e^{2} / h$, indicated in Fig. 6(b). The peaks split and re-form in a quasiperiodic fashion that does not seem to be directly related to the Fabry-Pérot oscillation period. They are also significantly narrower than even the de Gennes-Saint-James resonances, with source-drain widths of $\Delta V \approx 50-60 \mu \mathrm{V}$ according to FWHM. In Fig. 7(a), we detail the field evolution of one of these zero-bias anomalies. We find that the zero-energy resonance forms at finite field and can persist for at least up to $120 \mathrm{mT}$. At elevated temperatures [Fig. 7(b)], we find these anomalies broaden and merge with the background due to a combination of thermal fluctuations and dephasing.

These peaks are notable because no such features are seen in these two samples at zero magnetic field. They appear only when large fields are applied perpendicular to the TI surface and are accompanied by a collapse of the minigap governed by the Thouless energy [depicted in Fig. 7(a)]. The low-energy peaks do not appear when the TI flake is subjected to an in-plane magnetic field. Thus, they share many of the properties of zero-bias
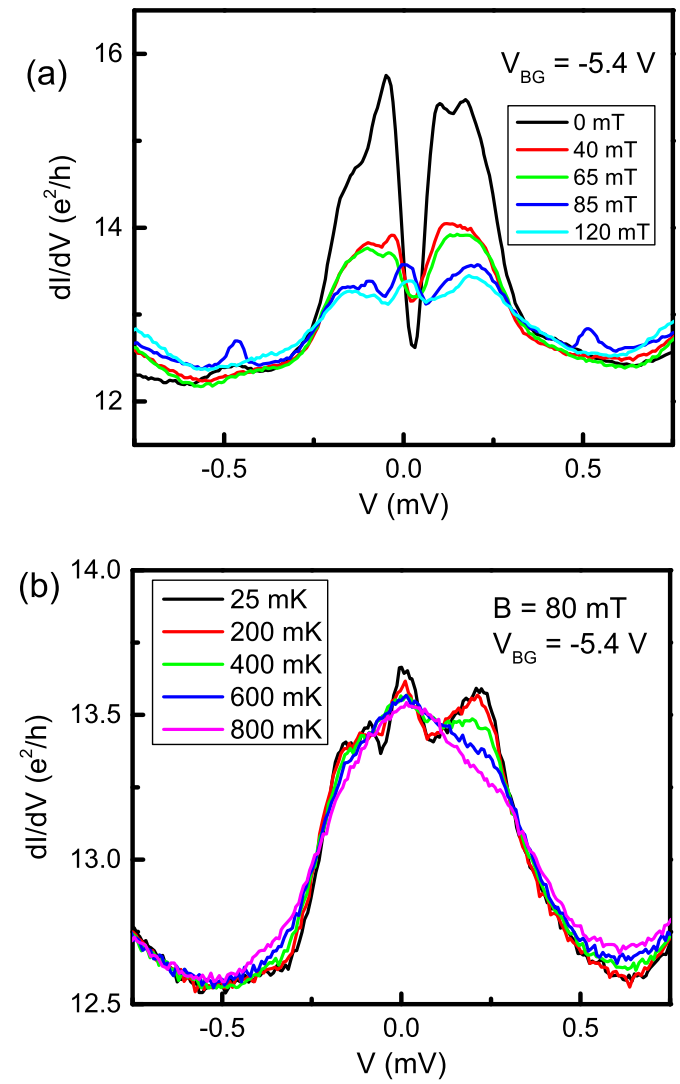

FIG. 7. (a) Magnetic-field evolution of zero-bias peak at $V_{\mathrm{BG}}=-5.4 \mathrm{~V}$. Peak appears beyond $65 \mathrm{mT}$ and remains for up to $\mathrm{B}=120 \mathrm{mT}$. No such peak is present at zero field or when an in-plane field is applied perpendicular to current flow. (b) Temperature dependence of zero-bias peak at $80 \mathrm{mT}$. Thermal fluctuations cause the peak to broaden and merge with background.

anomalies observed in spectroscopic studies of nanowires coupled to superconducting leads [24-28]. While such studies were interpreted in terms of Majorana fermions, here we are more cautious. For example, weak antilocalization can also generate low-energy resonances due to coherence between electron-hole trajectories that repeatedly impinge upon the superconductor-semiconductor interface [55]. Although originally proposed to describe diffusive semiconductors, such trajectories can exist in ballistic cavities due to multiple reflections off the boundary opposite of the superconductor-semiconductor interface [56]. In this respect, this phenomenon is similar to the de Gennes-Saint-James resonances but is restricted to near zero energy.

It has been pointed out that weak antilocalization in superconductor-semiconductor devices could survive in the presence of broken time-reversal symmetry, leading to low-energy anomalies that are nearly identical in appearance to those attributed to Majorana fermions [29]. Such lowenergy peaks can emerge even in topologically trivial materials. The persistence of de Gennes-Saint-James 
resonances and Fabry-Pérot oscillations [Figs. 5(a) and 5(b)] in our devices with magnetic field strongly suggests that the analogous trajectories for weak antilocalization can also remain phase coherent. This is in contrast to the previous assumption in the nanowire experiments that the large magnetic field would suppress weak antilocalization [24-28]. We note that while Refs. [57,58] explored nontopological origins of zero-bias anomalies in quantum dots coupled to superconducting leads, here we extend the experimental analysis to the limit of both ballistic transport and large coupling between the semiconductor segment and the metallic lead.

While the resonances in Fig. 6(a) might represent precursors to the low-energy Andreev bound states that are predicted to form around the vortices of topological superconductors [6], further work is required to confirm the existence of Majorana bound states. Fortunately, our results demonstrate that it is feasible to construct Fabry-Pérot interferometers on the surface of a topological insulator [9-11]. By flowing current through chiral edge channels around the superconducting lead, one could conceivably probe the $Z_{2}$ interference of paths that enclose an even or odd number of vortices with Majorana fermions. Such chiral edge channels will likely require inducing magnetization domains with ferromagnetic leads, which is beyond the subject of this present work.

\section{CONCLUSIONS}

We present transport measurements of the TI $\mathrm{Bi}_{2} \mathrm{Se}_{3}$ in contact with both a superconducting lead and a normal metal lead. At low temperature and zero magnetic field, we find evidence of both Andreev reflection and the reentrant resistance effect, confirming transparent contact between the TI and the superconductor. By tuning the chemical potential with electrostatic gates, we observe clear Fabry-Pérot oscillations with peak-to-peak amplitude $\approx 0.2 e^{2} / h$. For source-drain biases below the energy gap of the superconducting lead, the Fabry-Pérot oscillations double in frequency, demonstrating the emergence of de Gennes-Saint-James resonances. In relatively large magnetic fields, a number of low-energy peaks are observed, which mimic the previously reported signatures of MBSs but we strongly caution that they can also be explained in terms of weak antilocalization. Our findings show an interplay between phase-coherent transport and Andreev reflection. With a modified sample geometry, one can pursue interferometric searches of MBSs in TIs contacted with superconducting films. The robustness of the Fabry-Pérot oscillations despite multiple nanofabrication steps suggests that $\mathrm{Bi}_{2} \mathrm{Se}_{3}$ is a promising platform for more general studies of phase coherence in solid-state systems. Further study is needed to elucidate the role that topological surface states play in the Fabry-Pérot oscillations.

\section{ACKNOWLEDGEMENTS}

We acknowledge helpful discussions with Rudro Biswas, Fiona Burnell, Liang Fu, Pouyan Ghaemi, Taylor Hughes, and Shu-Ping Lee. C.K., A. D. K. F., and D. J. V.H. acknowledge funding by Microsoft Project Q. Y. S. H. acknowledges support from National Science Foundation Grant No. DMR-12-55607. Device fabrication was carried out in the MRL Central Facilities (partially supported by the U.S. DOE under DE-FG0207ER46453 and DE-FG02-07ER46471).

[1] W. Liang, M. Bockrath, D. Bozovic, J. H. Hafner, M. Tinkham, and H. Park, Fabry-Perot Interference in a Nanotube Electron Waveguide, Nature (London) 411, 665 (2001).

[2] A. V. Kretinin, R. Popovitz-Biro, D. Mahalu, and H. Shtrikman, Multimode Fabry-Perot Conductance Oscillations in Suspended Stacking-Faults-Free InAs Nanowires, Nano Lett. 10, 3439 (2010).

[3] F. Miao, S. Wijeratne, Y. Zhang, U. C. Coskun, W. Bao, and C. N. Lau, Phase-Coherent Transport in Graphene Quantum Billiards, Science 317, 1530 (2007).

[4] M.Z. Hasan and C. L. Kane, Colloquium:Topological insulators, Rev. Mod. Phys. 82, 3045 (2010).

[5] C. Nayak, S. H. Simon, A. Stern, M. Freedman, and S. Das Sarma, Non-Abelian Anyons and Topological Quantum Computation, Rev. Mod. Phys. 80, 1083 (2008).

[6] L. Fu and C. L. Kane, Superconducting Proximity Effect and Majorana Fermions at the Surface of a Topological Insulator, Phys. Rev. Lett. 100, 096407 (2008).

[7] J. Alicea, New Directions in the Pursuit of Majorana Fermions in Solid State Systems, Rep. Prog. Phys. 75, 076501 (2012).

[8] C. W. J. Beenakker, Search for Majorana Fermions in Superconductors, Annu. Rev. Condens. Matter Phys. 4, 113 (2013).

[9] L. Fu and C. L. Kane, Probing Neutral Majorana Fermion Edge Modes with Charge Transport, Phys. Rev. Lett. 102, 216403 (2009).

[10] A. R. Akhmerov, J. Nilsson, and C. W. J. Beenakker, Electrically Detected Interferometry of Majorana Fermions in a Topological Insulator, Phys. Rev. Lett. 102, 216404 (2009).

[11] K. T. Law, P. A. Lee, and T. K. Ng, Majorana Fermion Induced Resonant Andreev Reflection, Phys. Rev. Lett. 103, 237001 (2009).

[12] D. Zhang, J. Wang, A. M. DaSilva, J. Sue Lee, H. R. Gutierrez, M. H. W. Chan, J. Jain, and N. Samarth, Superconducting Proximity Effect and Possible Evidence for Pearl Vortices in a Candidate Topological Insulator, Phys. Rev. B 84, 165120 (2011).

[13] B. Sacépé, J. B. Oostinga, J. Li, A. Ubaldini, N. J. G. Couto, E. Giannini, and A. F. Morpurgo, Gate-Tuned Normal and Superconducting Transport at the Surface of a Topological Insulator, Nat. Commun. 2, 575 (2011).

[14] F. Qu, F. Yang, J. Shen, Y. Ding, J. Chen, Z. Ji, G. Liu, J. Fan, X. Jing, C. Yang, and L. Lu, Strong Superconducting 
Proximity Effect in $\mathrm{Pb}-\mathrm{Bi}_{2} \mathrm{Te}_{3}$ Hybrid Structures, Sci. Rep. 2, 339 (2012).

[15] M. Veldhorst, M. Snelder, M. Hoek, T. Gang, V. K. Guduru, X. L. Wang, U. Zeitler, W. G. van der Wiel, A. A. Golubov, H. Hilgenkamp, and A. Brinkman, Josephson Supercurrent through a Topological Insulator Surface State, Nat. Mater. 11, 417 (2012).

[16] S. Cho, B. Dellabetta, A. Yang, J. Schneeloch, Z. Xu, T. Valla, G. Gu, M. J. Gilbert, and N. Mason, Symmetry Protected Josephson Supercurrents in Three-Dimensional Topological Insulators, Nat. Commun. 4, 1689 (2013).

[17] J. B. Oostinga, L. Maier, P. Schüffelgen, D. Knott, C. Ames, C. Brüne, G. Tkachov, H. Buhmann, and L. W. Molenkamp, Josephson Supercurrent through the Topological Surface States of Strained Bulk HgTe, Phys. Rev. X 3, 021007 (2013).

[18] V. Orlyanchik, M. P. Stehno, C. D. Nugroho, P. Ghaemi, M. Brahlek, N. Koirala, S. Oh, and D. J. Van Harlingen, Signature of a Topological Phase Transition in the Josephson Supercurrent through a Topological Insulator, arXiv:1309.0163.

[19] C. Kurter, A. D. K. Finck, Y. San Hor, and D. J. Van Harlingen, Evidence for an Anomalous Current-Phase Relation of a dc SQUID with Tunable Topological Junctions, arXiv:1307.7764.

[20] L. Galletti, S. Charpentier, M. Iavarone, P. Lucignano, D. Massarotti, R. Arpaia, Y. Suzuki, K. Kadowaki, T. Bauch, A. Tagliacozzo, F. Tafuri, and F. Lombardi, Influence of Topological Edge States on the Properties of $\mathrm{Al} / \mathrm{Bi}_{2} \mathrm{Se}_{3} / \mathrm{Al}$ Hybrid Josephson Devices, Phys. Rev. B 89, 134512 (2014).

[21] C. Kurter, A. D. K. Finck, P. Ghaemi, Y. S. Hor, and D. J. Van Harlingen, Dynamical Gate-Tunable Supercurrents in Topological Josephson Junctions, Phys. Rev. B 90, 014501 (2014).

[22] P. G. de Gennes and D. Saint-James, Elementary Excitations in the Vicinity of a Normal Metal-Superconducting Metal Contact, Phys. Lett. 4, 151 (1963).

[23] J. M. Rowell and W. L. McMillan, Electron Interference in a Normal Metal Induced by Superconducting Contracts, Phys. Rev. Lett. 16, 453 (1966).

[24] V. Mourik, K. Zuo, S. M. Frolov, S. R. Plissard, E. P. A. M. Bakkers, and L. P. Kouwenhoven, Signatures of Majorana Fermions in Hybrid Superconductor-Semiconductor Nanowire Devices, Science 336, 1003 (2012).

[25] M. T. Deng, C. L. Yu, G. Y. Huang, M. Larsson, P. Caroff, and H. Q. Xu, Anomalous Zero-Bias Conductance Peak in a Nb-InSb Nanowire-Hybrid Device, Nano Lett. 12, 6414 (2012).

[26] A. Das, Y. Ronen, Y. Most, Y. Oreg, M. Heiblum, and H. Shtrikman, Zero-Bias Peaks and Splitting in an Al-InAs Nanowire Topological Superconductor as a Signature of Majorana Fermions, Nat. Phys. 8, 887 (2012).

[27] A. D. K. Finck, D. J. Van Harlingen, P. K. Mohseni, K. Jung, and X. Li, Anomalous Modulation of a Zero-Bias Peak in a Hybrid Nanowire-Superconductor Device, Phys. Rev. Lett. 110, 126406 (2013).

[28] H. O. H. Churchill, V. Fatemi, K. Grove-Rasmussen, M. T. Deng, P. Caroff, H. Q. Xu, and C. M. Marcus, Superconductor-Nanowire Devices from Tunneling to the
Multichannel Regime: Zero-Bias Oscillations and Magnetoconductance Crossover, Phys. Rev. B 87, 241401 (2013).

[29] D. I. Pikulin, J. P. Dahlhaus, M. Wimmer, H. Schomerus, and C. W. J. Beenakker, A Zero-Voltage Conductance Peak from Weak Antilocalization in a Majorana Nanowire, New J. Phys. 14, 125011 (2012).

[30] See Supplemental Material at http://link.aps.org/ supplemental/10.1103/PhysRevX.4.041022 for data from additional samples and additional micrographs of the main sample.

[31] J. G. Analytis, R. D. McDonald, S. C. Riggs, J.-H. Chu, G. S. Boebinger, and I. R. Fisher, Two-Dimensional Surface State in the Quantum Limit of a Topological Insulator, Nat. Phys. 6, 960 (2010).

[32] G. E. Blonder, M. Tinkham, and T. M. Klapwijk, Transition from Metallic to Tunneling Regimes in Superconducting Microconstrictions: Excess Current, Charge Imbalance, and Supercurrent Conversion, Phys. Rev. B 25, 4515 (1982).

[33] F. Yang, Y. Ding, F. Qu, J. Shen, J. Chen, Z. Wei, Z. Ji, G. Liu, J. Fan, C. Yang, T. Xiang, and L. Lu, Proximity Effect at Superconducting $\mathrm{Sn}-\mathrm{Bi}_{2} \mathrm{Se}_{3}$ Interface, Phys. Rev. B 85, 104508 (2012).

[34] G. Koren and T. Kirzhner, Zero-Energy Bound States in Tunneling Conductance Spectra at the Interface of an $s$-Wave Superconductor and a Topological Insulator in $\mathrm{NbN} / \mathrm{Bi}_{2} \mathrm{Se}_{3} / \mathrm{Au}$ Thin-Film Junctions, Phys. Rev. B 86, 144508 (2012).

[35] S. N. Artemenko, A. F. Volkov, and A. V. Zaitsev, On the Excess Current in Microbridges $s-c-s$ and $s-c-n$, Solid State Commun. 30, 771 (1979).

[36] Y. V. Nazarov and T. H. Stoof, Diffusive Conductors as Andreev Interferometers, Phys. Rev. Lett. 76, 823 (1996).

[37] A. A. Golubov, F. K. Wilhelm, and A. D. Zaikin, Coherent Charge Transport in Metallic Proximity Structures, Phys. Rev. B 55, 1123 (1997).

[38] T. H. Hsieh and L. Fu, Majorana Fermions and Exotic Surface Andreev Bound States in Topological Superconductors: Application to $\mathrm{Cu}_{x} \mathrm{Bi}_{2} \mathrm{Se}_{3}$, Phys. Rev. Lett. 108, 107005 (2012).

[39] S. Sasaki, M. Kriener, K. Segawa, K. Yada, Y. Tanaka, M. Sato, and Y. Ando, Topological Superconductivity in $\mathrm{Cu}_{x} \mathrm{Bi}_{2} \mathrm{Se}_{3}$, Phys. Rev. Lett. 107, 217001 (2011).

[40] J. G. Checkelsky, Y. S. Hor, R. J. Cava, and N. P. Ong, Bulk Band Gap and Surface State Conduction Observed in Voltage-Tuned Crystals of the Topological Insulator $\mathrm{Bi}_{2} \mathrm{Se}_{3}$, Phys. Rev. Lett. 106, 196801 (2011).

[41] D. Kim, P. Syers, N. P. Butch, J. Paglione, and M. S. Fuhrer, Coherent Topological Transport on the Surface of $\mathrm{Bi}_{2} \mathrm{Se}_{3}$, Nat. Commun. 4, 2040 (2013).

[42] K. I. Bolotin, K. J. Sikes, Z. Jiang, M. Klima, G. Fudenberg, J. Hone, P. Kim, and H. L. Stormer, Ultrahigh Electron Mobility in Suspended Graphene, Solid State Commun. 146, 351 (2008).

[43] M. Bianchi, D. Guan, S. Bao, J. Mi, B. Brummerstedt Iversen, P. D. C. King, and P. Hofmann, Coexistence of the Topological State and a Two-Dimensional Electron Gas on the Surface of $\mathrm{Bi}_{2} \mathrm{Se}_{3}$, Nat. Commun. 1, 128 (2010). 
[44] N. Bansal, Y. Seung Kim, M. Brahlek, E. Edrey, and S. Oh, Thickness-Independent Transport Channels in Topological Insulator $\mathrm{Bi}_{2} \mathrm{Se}_{3}$ Thin Films, Phys. Rev. Lett. 109, 116804 (2012).

[45] J. Cao, Q. Wang, and H. Dai, Electron Transport in Very Clean, As-Grown Suspended Carbon Nanotubes, Nat. Mater. 4, 745 (2005).

[46] F. Giazotto, P. Pingue, F. Beltram, M. Lazzarino, D. Orani, S. Rubini, and A. Franciosi, Resonant Transport in $\mathrm{Nb} / \mathrm{GaAs} / \mathrm{AlGaAs}$ Heterostructures: Realization of the de Gennes-Saint-James Model, Phys. Rev. Lett. 87, 216808 (2001).

[47] Y. Levi, O. Millo, N. D. Rizzo, D. E. Prober, and L. R. Motowidlo, Tunneling Spectroscopy of Bound and Resonant States in Superconducting Proximity Structures, Appl. Surf. Sci. 144-145, 575 (1999).

[48] J. P. Bird, K. Ishibashi, D. K. Ferry, Y. Ochiai, Y. Aoyagi, and T. Sugano, Phase Breaking in Ballistic Quantum Dots: Transition from Two-to Zero-Dimensional Behavior, Phys. Rev. B 51, 18037 (1995).

[49] A. G. Huibers, M. Switkes, C. M. Marcus, K. Campman, and A.C. Gossard, Dephasing in Open Quantum Dots, Phys. Rev. Lett. 81, 200 (1998).

[50] A. E. Hansen, A. Kristensen, S. Pedersen, C. B. Sørensen, and P. E. Lindelof, Mesoscopic Decoherence in AharonovBohm Rings, Phys. Rev. B 64, 045327 (2001).

[51] K.-T. Lin, Y. Lin, C. C. Chi, J. C. Chen, T. Ueda, and S. Komiyama, Temperature- and Current-Dependent Dephasing in an Aharonov-Bohm Ring, Phys. Rev. B 81, 035312 (2010).
[52] L. Bao, L. He, N. Meyer, X. Kou, P. Zhang, Z.-g. Chen, A. V. Fedorov, J. Zou, T. M. Riedemann, T. A. Lograsso, K. L. Wang, G. Tuttle, and F. Xiu, Weak Anti-localization and Quantum Oscillations of Surface States in Topological Insulator $\mathrm{Bi}_{2} \mathrm{Se}_{2} \mathrm{Te}$, Sci. Rep. 2, 726 (2012).

[53] D. Kim, S. Cho, N. P. Butch, P. Syers, K. Kirshenbaum, S. Adam, J. Paglione, and M. S. Fuhrer, Surface Conduction of Topological Dirac Electrons in Bulk Insulating $\mathrm{Bi}_{2} \mathrm{Se}_{3}$, Nat. Phys. 8, 460 (2012).

[54] A. V. Shytov, M. S. Rudner, and L. S. Levitov, Klein Backscattering and Fabry-Pérot Interference in Graphene Heterojunctions, Phys. Rev. Lett. 101, 156804 (2008).

[55] B. J. van Wees, P. de Vries, P. Magnée, and T. M. Klapwijk, Excess Conductance of Superconductor-Semiconductor Interfaces due to Phase Conjugation between Electrons and Holes, Phys. Rev. Lett. 69, 510 (1992).

[56] M. Schechter, Y. Imry, and Y. Levinson, Reflectionless Tunneling in Ballistic Normal-Metal-Superconductor Junctions, Phys. Rev. B 64, 224513 (2001).

[57] E. J. H. Lee, X. Jiang, R. Aguado, G. Katsaros, C. M. Lieber, and S. De Franceschi, Zero-Bias Anomaly in a Nanowire Quantum Dot Coupled to Superconductors, Phys. Rev. Lett. 109, 186802 (2012).

[58] E. J. H. Lee, X. Jiang, M. Houzet, R. Aguardo, C. M. Lieber, and S. De Franceschi, Spin-Resolved Andreev Levels and Parity Crossings in Hybrid Superconductor-Semiconductor Nanostructures, Nat. Nanotechnol. 9, 79 (2014). 